\title{
El impacto del modelo educativo dual en la formación profesional del estudiante
}

The Impact of the Dual Educational Model on the Student's Professional Training

O impacto do modelo educacional dual na formação profissional do aluno

Gustavo Geovanni Flores-Sánchez* (iD) hteps://orcid.org/0000-0003-4123-2644

Hernán Pedro Vigier** (iD htrps://orcidorg/0000-0003-0774-8620

\section{Doi: 10.17227/rce.num78-9535}

Para citar este artículo: Flores Sánchez, G., y Vigier, H. (2020). El impacto del modelo educativo dual en la formación profesional del estudiante. Revista Colombiana de Educación, 78, 173-205. http://doi. org/10.17227/rce.num78-9535

\section{(C) $(1) \Theta$}

* Doctor en Ciencias de la Administración. Profesor - Investigador del Grupo de Investigación Empresarial - GIE de la Facultad de Ciencias Económicas y Administrativas en la Universidad de Cuenca, Ecuador. Correo electrónico: gustavo.flores@ucuenca.edu.ec

** Doctor en Dirección y Administración de Empresas. Profesor del Departamento de Economía en la Universidad Nacional del Sur. Correo electrónico: hvigier@uns.edu.ar 


\section{Resumen}

Esta investigación tiene el objetivo de estudiar el impacto de la formación dual, una modalidad de innovación educativa de enseñanza y aprendizaje que se desarrolla en dos lugares distintos y complementarios (el aula y la empresa), en la formación de los profesionales y su inserción en el mercado laboral. El estudio asume un corte transversal cuasiexperimental. Para la estimación del impacto se usó el propensity score matching con tres algoritmos: vecino más cercano, radio y el kernel matching y, por otro lado, el análisis de puntuaciones de regresión. Los resultados son sólidos tanto en estos dos enfoques, como en los tres algoritmos. Como contribución se evidenció que los egresados que participan en el programa dual tienen un salario significativamente mayor (USD 275.00 USD) y un menor tiempo (2 meses 20 días) en insertarse en el mercado laboral con respecto a los que no recibieron el programa (modelo tradicional).

\section{Palabras clave}

educación profesional; educación no tradicional innovación educativa; escuelas tradicionales: administración de negocios

\section{Keywords}

professional education; nontraditional education; educational innovation; traditional schools; business administration

\begin{abstract}
This research has the objective of studying the impact of dual training, a form of educational innovation of teaching and learning that takes place in two different and complementary places (the classroom and the company), in the training of professionals and their insertion in the labor market. This study assumes a quasi-experimental cross section. For the estimation of the impact the Propensity Score Matching was used, which also uses three algorithms: the nearest neighbor, the radio and the kernel matching, and, the analysis of regression scores as well. The two approaches: Propensity Score Matching and regression score analysis, as in the three algorithms, give solid results as a contribution it was evidenced that the graduates who participate in the dual program have a significantly higher salary (\$275.00 USD) and a shorter time (2 months 20 days) in inserting in the labor market with respect to those who did not receive the program (Traditional model).
\end{abstract}

\section{Resumo}

Esta pesquisa tem como objetivo estudar o impacto do treinamento duplo, uma modalidade de inovação educacional no ensino e aprendizagem que ocorre em dois locais diferentes e complementares (sala de aula e empresa), na formação de profissionais e sua inserção no mercado de trabalho. O estudo é de tipo quase experimental. Para a estimativa do impacto, utilizou-se o propensity score matching, com três algoritmos vizinho mais próximo, rádio e kernel matching e, por outro lado, a análise de escores de regressão. Os resultados são sólidos nas duas abordagens e nos três algoritmos. Como contribuição, ficou evidenciado que os graduados que participam do programa duplo têm um salário significativamente mais alto (US \$275,00) e menos tempo (2 meses e 20 dias) para entrar no mercado de trabalho em comparação com aqueles que não receberam o programa (modelo tradicional).

\section{Palavras-chave}

educação profissional; educação não tradicional inovação educacional; escolas tradicionais; administração de empresas 


\section{Introducción}

Para autores como Alemán (2015) y Velázquez Albo (2017), la educación tradicional universitaria ha representado un procedimiento formal en el que un estudiante cumple con una serie de requisitos para obtener un título al final de una carrera, título que acredita que puede desempeñarse en un puesto de trabajo. El problema con ese proceso es que excluye elementos fundamentales de la educación como el aprendizaje significativo, tareas autónomas de enseñanza dirigidas a los estudiantes, así como las sinergias que ocurren en el mundo empresarial.

Gómez (2017) definió la educación como un proceso de socialización y endoculturación de las personas, a través del cual se desarrollan capacidades físicas e intelectuales, habilidades, destrezas, técnicas de estudio y formas de comportamiento ordenadas con un fin social. Sin embargo, como Gessler (2017) demostró, las nuevas exigencias del mercado en términos de aptitudes laborales han obligado a las universidades a complementar esa formación para establecer una estrecha relación entre la educación académica y la experiencia. Surge así la necesidad de profesionalizar a los estudiantes para responder a las nuevas exigencias de los sistemas productivos.

En tal virtud, lo explicaron Marhuenda-Luixá, Chisvert-Tarazona, Palomares-Montero y Vila (2017), el modelo dual de enseñanza en la universidad emergió como una forma de combinar el aprendizaje teórico del aula con las prácticas en las empresas, para que así se potencializaran las capacidades de los estudiantes y respondieran a necesidades empresariales concretas; como resultado, se mejoran las condiciones laborales tanto en empleabilidad como en términos económicos. Esta iniciativa nació en Europa y se institucionalizó en Alemania como pilar de los sistemas de educación y formación profesional cuyo objetivo fue reducir el desempleo juvenil y mejorar la empleabilidad (Kenzhegaliyeva, 2018).

Varios países latinoamericanos han implementado la formación dual. En Ecuador, esta opera desde 2006. La Universidad de Cuenca, en Cuenca, adoptó esta estrategia educacional basada en el sistema de alternancia entre universidad y empresa con el objetivo de preparar a los jóvenes para su desempeño en el mundo laboral y la continuidad de sus estudios sistemáticos. Pero, a pesar de la concentración de experiencias desarrolladas en Latinoamérica al respecto, como argumentó Kupffer (2015), existen pocos estudios que vinculen esta estrategia con la educación universitaria.

En esta investigación, considerando lo dicho, se pretende sistematizar y difundir el impacto del modelo dual universitario en Ecuador en la inserción laboral como elemento fundamental que vincula el mundo académico y el trabajo. Para el efecto, se tomó como caso de estudio la formación dual de la Universidad de Cuenca en el periodo 2010-2016, 
que empleó como enfoques un emparejamiento de puntajes de propensión que aplica tres algoritmos y un análisis de puntuaciones de regresión que buscaba evidenciar si había diferencias significativas en los salarios y el tiempo que se toman en insertarse al mercado laboral los egresados de la dual (grupo de tratamiento), con respecto a los egresados de la formación tradicional (grupo de control).

\section{Marco conceptual}

Actualmente, las empresas y organizaciones avanzan gracias a la interacción, difusión, aplicación y sistematización de un conocimiento creado u obtenido. Para Villarroel, Cabrales, Fernández y Godoy (2017), el proceso de aprendizaje organizacional se da a través de las relaciones interinstitucionales entre el gobierno, las empresas y las universidades, lo que genera desarrollo y competitividad.

De igual forma, en la economía del saber se reconoce el rol de la educación superior como productora y difusora del conocimiento, como base de la competitividad de los territorios y países. Asimismo, como bien observaron Carranza, Acosta y Pulido (2016), las universidades están llamadas a actuar como vínculo entre el estudiante y el mundo laboral; en ese sentido, su propósito es ejecutar planes y acciones en innovación, cultura, prácticas profesionales, intercambio de tecnología, entre otros. Este proceso se ha cristalizado mediante instrumentos de colaboración que han dado como fruto un beneficio mutuo en la mejora de los servicios y un incremento de la productividad. Cordero, Muniz y Simancas (2017) explicaron que la vinculación entre las universidades y las empresas se da en una forma de cooperación mutua, basada en acuerdos cuyo beneficio consiste tanto en la transferencia de información y tecnología, como en la ejecución de acciones integrales que permitan el crecimiento y desarrollo de las personas e instituciones involucradas.

En este sentido, Pineda-Herrero, Argud-Morell y Ciraso-Calí, (2016) han considerado que las actuales necesidades del sector empresarial y la sociedad exigen a los alumnos universitarios adquirir habilidades, conocimientos y destrezas que les faculten ser competitivos e innovadores. Por esto, las universidades deberían proponer su oferta educativa y académica, y definir la pertinencia de sus carreras de forma que puedan proporcionar capital humano con mayores probabilidades de obtener puestos profesionales, con alto nivel salarial, acordes con las necesidades del mercado para crear así una mejor relación entre educación, capital humano y mercado laboral.

De esta forma Bentolila, Cabrales y Jansen (2018) delinean la necesidad de que los estudiantes universitarios combinen la formación general que reciben en las aulas con habilidades y destrezas específicas (proporcionadas 
por el trabajo), con el propósito de generar profesionistas con mayores competencias, que contribuirán a reducir los tiempos de inserción y adaptación en el mercado laboral. De igual manera, se debe considerar que los procesos de inserción laboral de quienes egresan de la educación superior no dependen exclusivamente de la formación recibida, como señalaron Román, Franco y Camacho (2018), sino que también concurren diferentes factores determinantes como sociales, económicos e interpersonales definidos por la edad, sexo, procedencia, relacionamiento, competencias cognitivas y aspectos físicos, entre otros aspectos que condicionan las posibilidades de empleo e inserción laboral (Vera, 2015).

Esta relación entre empresa y universidad se puede vislumbrar en el sueldo que recibe un empleado. Becker (1975), al exponer el modelo de capital humano, admitió que existe una relación entre los sueldos, la escolaridad y la experiencia en el trabajo; con ello insta a estimar ecuaciones que relacionen los ingresos con los años de educación. De igual forma, Berniell y De la Mata (2017) demostraron que la teoría del capital humano permite entender el mercado laboral como el vínculo entre educación y economía, y señalaron que los ingresos de los individuos aumentan con el nivel de educación.

Otros estudios nos brindan resultados valiosos sobre estas relaciones entre universidad y empresa. Dang (2016), por ejemplo, analizó los efectos de la formación en educación vocacional (VET, por su sigla en inglés) en el salario de los empleados en el contexto vietnamita y comprobó que la capacitación en el trabajo se asoció positivamente con los aumentos salariales a través de una mejor competencia técnica; la educación formal se relacionó positivamente con el salario y, por último, apreció que las empresas que introdujeron más tecnología dieron lugar a que sus empleados fueran más eficientes, mejoraran su desempeño e incrementaran sus ingresos. Otro ejemplo son los estudios de Brunet y Rodríguez-Soler (2017), realizados en Francia y Suiza sobre la relación trabajo y estudios de quienes cursan niveles universitarios, los cuales mostraron que la experiencia laboral adquirida afecta positivamente en el futuro del estudiante cuando este se inmiscuye en el mercado laboral: a mayor experiencia menor probabilidad de estar desempleado y mayor posibilidad de incrementar su salario.

Asimismo, Orellana, Răileanu y Argudo (2016) investigaron los rendimientos de la educación en Ecuador y concluyeron que cuanto mayor es el nivel de educación mayor es la tasa de retorno: por cada año adicional de educación aumenta el rendimiento en casi un $6 \%$. Los resultados les permitieron inferir que la educación podría ser una de las medidas políticas más eficaces contra la pobreza y la desigualdad de ingresos. Vera (2015) observó fuertes recompensas cuando se obtienen títulos (sheepskin effect) en los niveles de educación superior, con excepción de la educación media técnica. En su evidencia, la instrucción universitaria afecta directamente

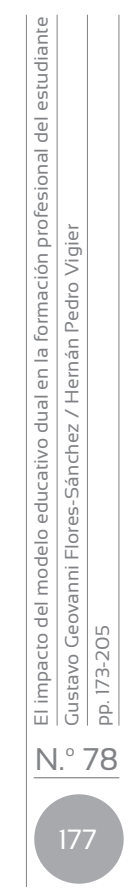


el aumento de la productividad (o capital humano) del individuo y su relación con el mercado laboral. El mejoramiento de la cantidad y calidad de la educación, entonces, se considera como una condición clave para la inserción laboral exitosa. También se deben considerar el estudio referido por Chávez, Guzmán, Rodríguez y Toledo (2017) sobre la actual situación de dependencia financiera de las mujeres en Latinoamérica para lograr la igualdad salarial, reducir la discriminación en el mercado laboral, y la decisión libre sobre su vida productiva y sexual. Los autores concluyeron que, pese a que se ha generado un marco normativo y cultural de inclusión de la mujer en las organizaciones ecuatorianas hoy en día, persisten los prejuicios, estereotipos y conductas discriminatorias en la inserción laboral de la mujer.

De forma más concreta, Zhibin y Weiping (2017) estimaron que un acercamiento más temprano al mundo laboral por parte de los estudiantes universitarios mediante las pasantías o una combinación de formación teórica y práctica podrían minimizar el impacto psicológico de esta transición y facilitar el desarrollo de la identidad personal. Esta práctica daría lugar a una inserción en el mercado laboral en el menor tiempo debido a las experiencias prácticas adquiridas y validadas en el sector productivo. De igual manera, Dang (2016) explicó que el vínculo entre la formación universitaria y el empleo puede describirse como parte de una meritocracia educacional: cuanto mayor es el nivel alcanzado por la persona al final de su educación previa al trabajo más probabilidades de éxito tendrá en el mundo laboral, como un buen sueldo, estatus, poder e influencia social.

Considerando estos valiosos aportes, Rivera (2017) propuso que una exigencia y requisito de políticas y lineamientos institucionales debería ser la vinculación de las universidades con el mundo laboral. Con esta premisa emergió la educación dual como una modalidad de enseñanza y aprendizaje que se lleva a cabo en dos lugares combinados y complementarios: el centro universitario y la empresa. Para Medina y Paniagua (2017), el principio fundamental de este modelo pedagógico lo constituye la relación educación/trabajo, mediante un proceso enseñanza/aprendizaje en dos lugares con una lógica cooperativa, fortalece la formación del capital humano capaz de incidir en la productividad de las empresas y en el desarrollo económico del territorio. Pilz (2016) ha señalado que la modalidad dual universitaria es una promesa académica innovadora que busca la conveniencia entre el tejido productivo, los centros de educación y los estudiantes, y producirá sinergias de reciprocidad diferentes del modelo tradicional universitario en donde coexiste una formación académica netamente en aula con una baja interacción con la empresa. Martín et al. (2017) y Yahaya, Sattar y Mohamad (2017) han determinado que la formación dual en los centros educativos superiores está orientada 
a un proceso educativo integral, en el cual el estudiante alcanza un nivel de desarrollo en un puesto de trabajo que le ayudará a competir como un profesional altamente calificado por sus cualidades humanas, intelectuales, prácticas y actitudinales.

El programa curricular de la formación dual en la universidad regula que los estudiantes deben cumplir un $50 \%$ del tiempo de permanencia en cada fase. En la etapa de formación o aula, el estudiante adquiere los conocimientos teóricos y científicos sobre su profesión, así como las competencias cognitivas requeridas para la carrera, mientras que en la fase de prácticas en la empresa puede integrarse mediante diversas formas e intensidades que obedece a un plan de rotación en el que se definen las funciones que el estudiante cumplirá (Alemán, 2015; Cordero, Muniz y Simancas, 2017).

Las ventajas competitivas del sistema de educación dual en América Latina también han sido comprobadas. Rojas (2015) evidenció que el modelo potenció la vinculación laboral de los estudiantes universitarios de la Fundación Empresarial de la Cámara de Comercio de Bogotá, y puso en relieve su satisfacción laboral medida por los ingresos, satisfacción en el trabajo y la implementación del proyecto en la empresa, un factor relevante y significativo en la formación dual. De igual forma, Segura (2016), en su estudio de la medición de impacto de la política de formación dual universitaria en Colombia, verificó el valor del contrato de aprendizaje (CA) como una estrategia de formación para el trabajo que combina adiestramiento en aspectos vocacionales con el desarrollo de una fase práctica en empresas y evidenció su ventaja: mejores perspectivas de ocupación y de ingresos para el trabajador. Herrera, Tinoco y Guzmán (2015) evidenciaron la gran calidad de la modalidad dual en la carrera de Ingeniería Electromecánica que ofrece el ITESI de México en función de los resultados académicos y laborales obtenidos por los estudiantes. El programa, además, ha supuesto la cooperación mutua entre la institución superior y la empresa formadora para instaurar proyectos de investigación y desarrollo tecnológico.

Entre los estudios centrados en la formación dual en el país podemos mencionar el efectuado por Carvajal, Romero y Álvarez (2017) acerca de la implementación del modelo dual en la Universidad de Técnica de Ambato-Ecuador. En sus conclusiones apuntan que esta formación no solo resultó de beneficio para el estudiante y la academia, sino que se ha convertido en una fortaleza para el sector empresarial que recibe una importante transferencia de conocimientos actualizados del estudiante, concretamente los adelantos de la ciencia que coadyuvan al perfeccionamiento de los procesos empresariales, lo que brinda una ventaja competitiva a la institución. 


\section{Método}

En la presente investigación se efectúa un análisis cuantitativo, descriptivo y comparativo de los salarios y el tiempo que toman los egresados de los dos programas en insertarse al mercado laboral. Así mismo, se emplea una técnica de tipo cuasiexperimental de corte transversal porque toma como estrategia de análisis un estudio de caso, el programa dual de la Universidad de Cuenca, con una serie de variables que marcan la diferencia con otros grupos. Estas variables se han definido considerando la propuesta de Lu, Cai y Tong (2018): la mejor forma de evaluar el impacto de un programa o proyecto educativo es estimar los cambios en el bienestar de los estudiantes y definir si esos efectos son atribuibles a la intervención del programa. Para el uso de esta técnica consideramos la propuesta de Caliendo y Kopeinig (2008); Nasif et al. (2017), y Ovalle (2015). Según su visión, la técnica cuasiexperimental del emparejamiento de puntajes de propensión en investigaciones educativas aporta ideas para solucionar temas de sesgos de selección y el inconveniente de llevar a cabo experimentos de orden ético y no aleatorio. Además, esta técnica se sustenta en parámetros estadísticos que arrojan resultados tan precisos como los hallados en situaciones experimentales.

Dentro de esta técnica cuasiexperimental, se considera $i$ el índice de la población de egresados bajo atención; $T_{i^{\prime}}$ una variable binaria igual a 1, si el $i$-ésimo egresado se le asigna el tratamiento (modelo dual) o si es asignado al grupo de control (modelo tradicional). Por tanto, $Y_{i 1}$ es el resultado potencial en los salarios que poseen y el tiempo que toma un egresado del modelo dual en insertarse al mercado laboral (1); $Y_{i 0}$ es el valor de las mismas variables cuando el egresado está en el programa tradicional (0). En este sentido, el efecto del tratamiento para un egresado es:

$$
\Delta_{i}=Y_{i 1}-Y_{i 0}
$$

Debido a que no se posee conocimiento de que el mecanismo de asignación de un estudiante para el ingreso al programa dual de la Universidad de Cuenca sea aleatorio (por ejemplo, si algunos estudiantes que quisieron estudiar en esta carrera lograron ser admitidos o si la asignación se hizo por orden de llegada o por la cercanía a la institución, entre otras) y debido a que se desea evitar alguna regla discrecional al seleccionar a los egresados, la presente investigación utiliza dos enfoques: el análisis de puntuaciones de regresión y el propensity score matching (PSM) para escoger al grupo de control (tradicional) y calcular el impacto promedio de la formación dual. 
En consecuencia, partiendo de esta premisa planteada, que la formación dual en la Universidad de Cuenca no consideró en su diseño la asignación aleatoria de sus estudiantes para el ingreso a la carrera, una de las soluciones para evaluar diferencias en los resultados de interés es tratar de simular la aleatoriedad en la asignación. En este sentido, Caliendo y Kopeinig (2008) y Kool et al. (2017) mencionaron que los métodos de emparejamiento como el PSM pretenden construir un grupo de comparación o control lo más similar posible en términos de unas características observables.

Bajo ciertas condiciones, Caliendo y Kopeinig (2008); Heinrich, Maffioli y Vázquez (2010); Failla, Melillo y Reichstein (2017) explicaron que esta técnica puede ser más adecuada para controlar variables observables que la de mínimos cuadrados, debido a que no supone una relación lineal entre los observables y la variable dependiente, y porque analiza si la distribución de los observables se superpone entre los dos grupos de comparación. Igualmente, Heinrich, Maffioli y Vázquez (2010), y Bodory, Camponovo, Huber y Lechner (2018) indicaron que en un contexto no experimental el efecto tratamiento relevante que se considerará es el efecto medio de tratamiento sobre los tratados (average treatment effect on the treated, ATET). En este caso, el efecto promedio de recibir el programa dual sobre los salarios o el tiempo de inserción en el mercado laboral de los egresados está representando por: Caliendo y Kopeinig (2008), Heinrich, Maffioli y Vázquez (2010), Heinrich et al. (2010) y

$$
\operatorname{ATET}=E\left[\Delta_{i} \mid T_{i}=1\right]=E\left[Y_{i 1} \mid T_{i}=1\right]-E\left[Y_{i 0} \mid T_{i}=1\right]
$$

En donde, dada una muestra de egresados, $E\left[Y_{i 1} \mid T_{i}=1\right]$ puede ser calculada, pero $E\left[Y_{i 0} \mid T_{i}=1\right]$ no es observada. Ante la ausencia de un grupo de control experimental, es posible utilizar un grupo de egresados potencialmente comparables siempre que presenten el mismo conjunto de variables observables $X_{i}$. En este caso, para lograr identificar el ATET, se asume que el resultado de no tratamiento $Y_{i 0}$ es independiente del tratamiento $T_{i}$ una vez condicionado en $X_{i}$; en donde:

$$
Y_{i 0}\left|T_{i}\right| X_{i}
$$

Este supuesto, según Imbens (2004), Imbens y Wooldridge (2009), y Ovalle (2015), se conoce como de no confusión (unconfoundedness), es decir, la asignación al tratamiento depende solo de las variables observables $X_{i}$ que se pueden controlar. Por tanto, una vez condicionadas estas últimas, 
en esta investigación no hay una diferencia sistémica antes del tratamiento entre los egresados asignados al modelo dual y los asignados al modelo tradicional; de esta manera, el ATEt se describiría de la siguiente manera:

$$
A T E T=E\left\{E\left[Y_{i 1} \mid T_{i}=1, X_{i}\right]-E\left[Y_{i 0} \mid T_{i}=0, X_{i}\right] \mid T_{i}=1\right\}
$$

En consecuencia, una primera forma de emparejar a los egresados del modelo dual con los del tradicional es mediante las características observables. Estos datos se estratifican en subgrupos, cada uno se define por un valor particular de $X$. Cuando esta variable toma muchos valores distintos se dificulta efectuar un emparejamiento, porque, según lo explican Imbens y Wooldridge (2009), y López, Virgüez, Sarmiento y Silva (2015), coexiste un problema de dimensionalidad. Por tanto, no es posible encontrar una pareja para los egresados del modelo dual para unos valores de $X$. Para afrontar este problema, Imbens (2004) y Failla, Melillo y Reichstein (2017) propusieron el emparejamiento mediante puntaje de propensión, empleando un modelo probit o logit en el caso de tratamiento binario, en donde $p\left(X_{i}\right)=P_{r}\left[T_{i}=1 \mid X_{j}\right]$ es la probabilidad condicional de que el individuo $i$ reciba tratamiento dado $X_{i}$ si o $<p\left(X_{i}\right)<1$, demostraron que [3] implican:

$$
Y_{i 0}\left|T_{i}\right| P_{r}\left(T_{i}=1 \mid X_{i}\right)
$$

Así, el supuesto de independencia [3] puede ampliarse para el puntaje de propensión y también para el cálculo del ATET:

$$
A T E T=E\left\{E\left[Y_{i 1} \mid T_{i}=1, p\left(X_{i}\right)\right]-E\left[Y_{i 0} \mid T_{i}=0, p\left(X_{i}\right)\right] \mid T_{i}=1\right\}
$$

El estimado ATET de emparejamiento utiliza el puntaje de propensión como un esquema de ponderación de las unidades de comparación:

$$
A T E T=\frac{1}{N} \sum_{i \in N}\left(Y_{i 1}-\sum_{j \in / i} \omega_{i j} Y_{O j}\right)
$$

En donde $N$ es el número de participantes; $\omega_{i j^{\prime}}$ el peso dado al egresado; $j$-ésimo del modelo tradicional al efectuar la comparación con el egresado; $i$-ésimo del modelo dual, $\sum_{j \in l i} \omega_{i j}=1$, del cual se pueden obtener diferentes estimadores de emparejamiento cambiando la elección de $\boldsymbol{\omega}_{i j}$.

Para este efecto, Caliendo y Kopeinig (2008); Heinrich, Maffioli y Vázquez (2010), Caliendo y Kopeinig (2008), Heinrich et al. (2010) y James et al. (2017) sugirieron tres técnicas o algoritmos de emparejamiento que 
se utilizan para experimentar la robustez y consistencia de los resultados de acuerdo con las especificaciones propuestas: el vecino más cercano, radio matching y kernel matching. La técnica del vecino más cercano consiste en escoger al egresado del modelo tradicional con el puntaje de propensión más próximo al de cada egresado del modelo dual. El emparejamiento de radio matching define la distancia máxima con la que se buscan las unidades de comparación. Con la técnica de kernel se consideran a todos los egresados del modelo tradicional, pero el peso de cada uno de ellos depende de su distancia con respecto al egresado del modelo dual. Dicho de otro modo, cualquier técnica que se utilice para comparar los salarios y el tiempo que se toman en insertarse al mercado laboral a los egresados del modelo dual y tradicional buscará utilizar variables observables para conformar los grupos, sabiendo que siempre existirán variables no observables que influyan tanto en los salarios como en el tiempo de demora en insertarse en el mercado laboral.

Heinrich, Maffioli y Vázquez (2010); Dehejia y Wahba (2016), y Wagner, Newman y Javitz (2017) señalaron que para que el PSM logre una buena estimación en impacto también debe cumplir con un supuesto de soporte común que garantiza que los participantes tanto de tratamiento como de control sean observados en situaciones de comparación semejantes a la distribución de las puntuaciones de propensión de las observaciones, de tal forma que una considerable región de soporte común pueda ser identificada, así:

$$
0<P_{r}\left(T_{i}=1 \mid X_{i}\right)<1
$$

Esta condición asegura que los individuos o egresados con los mismos valores de $X_{i}$ dispongan de una probabilidad efectiva de ser participantes como no participantes.

Asimismo, Imbens (2004), e Imbens y Wooldridge (2009) mencionan que un análisis de puntuación de regresión controla las diferencias en las características observadas entre los egresados del programa dual y tradicional. Así, el resultado de los salarios y el tiempo de inserción laboral esperado y condicional de los egresados está dado por un conjunto de covariables:

$$
E(Y \mid X, w)=\mu_{o}+\alpha_{w}+x_{\beta}+w(X-\bar{X}) Y
$$

Donde, $Y=(1-w) Y_{0}+w Y_{1}$ es el resultado observado $\left(Y_{1}\right.$ para los egresados dual $Y_{0}$ para la tradicional). Restando los valores promedios $\bar{X}$ de las covariables $X$ garantiza que el coeficiente $\alpha$ sea el efecto de 
tratamiento promedio. Dado que la regresión $Y, X, w(X-\bar{X})$ estima los parámetros de manera consistente; se deriva una estimación del efecto medio del tratamiento condicional:

$$
A T E T_{1}^{R e g}=E(\alpha+(X-\bar{X}) \gamma \mid W=1) \quad[10]
$$

\section{Muestra}

Para medir la contribución y el impacto de la formación dual en términos de salario e inserción laboral, como un factor vinculador entre el mundo académico y laboral en Ecuador, se tomó como estrategia de análisis el caso de estudio de los egresados de la Facultad de Ciencias Económicas y Administrativas de las carreras de Ingeniería Empresarial (programa dual) y Administración de Empresas (programa tradicional) de la Universidad de Cuenca por semejanzas en sus mallas curriculares. Para el efecto, se definió llevar a cabo un censo a los egresados de los mencionados programas en el periodo 2010-2016, debido a que el año 2010 egresó la primera promoción dual y 2016 al cumplirse una década de existencia de dicha formación. El universo considerado es de 474 egresados de las dos carreras: 140 del modelo dual y 334 del tradicional, es decir, todos los estudiantes que se graduaron en ambas modalidades.

Adicionalmente, se efectuaron 387 entrevistas, a un $86 \%$ (121) del número de egresados correspondientes a la formación la dual y un $80 \%$ (266) del número de egresados del modelo tradicional. No se pudo entrevistar al cien por ciento de los egresados por diversos factores: un $14 \%$ de la dual, debido a la desactualización de las direcciones del domicilio o del lugar de trabajo de los egresados, y $20 \%$ del modelo, por similares razones; un $87 \%$ tiene desactualizado la dirección de domicilio y sus datos de contacto, y un 13 \% está fuera del país.

De acuerdo con estas cifras, por inferencia estadística en cada grupo poseemos un nivel de confianza del 95 \%, con una población dual de 140 y un tamaño de muestra de 121, y obtenemos de error máximo admisible +/- 2,72 \%; de la misma forma con una población tradicional de 334 y un tamaño de muestra de 266, el error máximo admisible es de +/$3,3 \%$, considerando a las dos muestras de estudios representativas para la población de egresados.

\section{Instrumento}

Para llevar a cabo la investigación y obtener la información se utilizó un cuestionario de preguntas abiertas, cerradas y de elección múltiple, que proporcionara información y caracterizara a los egresados, tanto de 
la formación dual como de la tradicional, de la Universidad de Cuenca de los años 2010-2016. Para recabar la información del cuestionario se desarrollaron visitas personales y se usó el correo electrónico. También se recurrió a datos extraídos de los cuestionarios o fichas técnicas de admisión, aplicados a los estudiantes y padres de las dos carreras, datos proporcionados por la Unidad de Admisión y Becas de dicha institución, que registra información socioeconómica, demográfica y educativa relevante para el interés de la investigación.

\section{Variables}

Como se detalló anteriormente, las técnicas de emparejamiento de los puntajes de propensión requieren la definición de las variables $X_{i}$ de las características de tratamiento de los participantes considerando las condiciones que podrían incidir en la probabilidad de pertenecer o no al modelo dual. Entonces se decidió contar con cuatro grupos de variables; un grupo de variables de impacto e interés y los siguientes grupos, variables explicativas observadas.

En el primero grupo se considera la variable dicotómica de impacto que es la formación dual y como variables continuas de interés el salario mes y el tiempo en insertarse en el mercado laboral. Como segundo grupo se particularizan las características sociodemográficas y personales del egresado como género, estado civil, especialidad del bachillerato. El tercer grupo toma en cuenta las características del hogar del egresado como el tipo de vivienda, zona donde vive, lugar de procedencia. El cuarto grupo concentra las características del ámbito laboral, como el sector económico en el que labora, el cargo que desempeña y años de experiencia.

En lo referente al tratamiento de los datos categóricos de las variables explicativas $X_{i}$ como de la variable de impacto se generaron variables dummy (tantas como el número de categorías de la variable original menos una, siendo la categoría de referencia siempre la más frecuente).

\section{Resultados}

A continuación, se presentan los principales resultados estadísticos descriptivos y comparativos de los programas en mención. En la figura 1, se observa la participación de los egresados de los dos programas por género, sector y salario promedio de los años 2010 al 2016. Se aprecia que $58 \%$ de mujeres y $56 \%$ de hombres de la dual trabajan en el sector de servicios, frente a un $50 \%$ de los egresados tanto mujeres como hombres de la tradicional. Así mismo, el salario promedio de los egresados hombres y 
mujeres de la dual que laboran en el sector servicios es de USD 1283,50 y US 1011, en comparación a los egresados de la tradicional que es de USD 623,96 y USD 680,32 respectivamente.

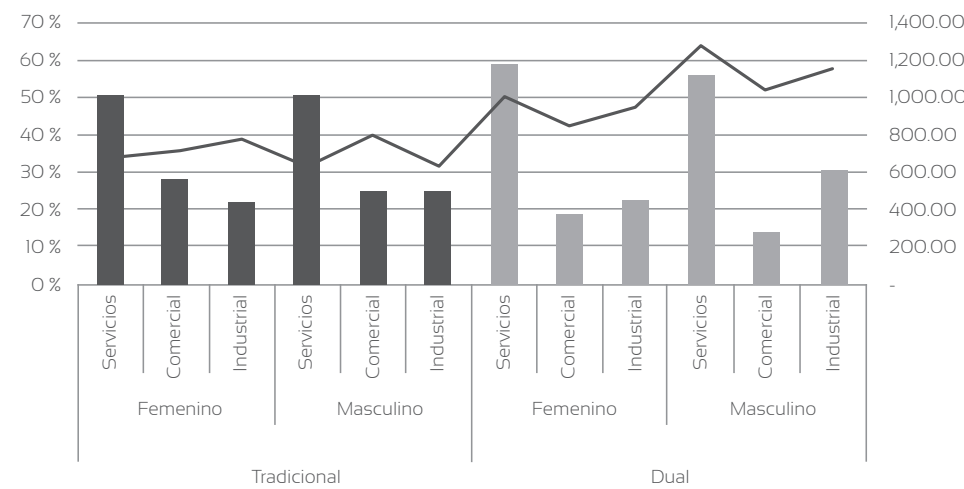

Figura 1. Ocupación de los egresados del sistema dual y tradicional de la Universidad de Cuenca, por género, sector y salario promedio en el periodo 2010-2016

Fuente: elaboración propia.

En la figura 2, se evalúan los salarios de los egresados de la formación dual y tradicional, considerando los cargos que desempeñan y los años de experiencia laborales. De esta manera se observa que los egresados de la dual poseen más años de experiencia y desempeñan cargos de jerarquía como gerentes, directores y coordinadores con un salario medio de USD 1253, que representa un $23 \%$ más frente a los egresados de la tradicional. Así mismo, se aprecia un $62 \%$ y $40 \%$ más en los salarios de asistentes y analistas del programa dual.

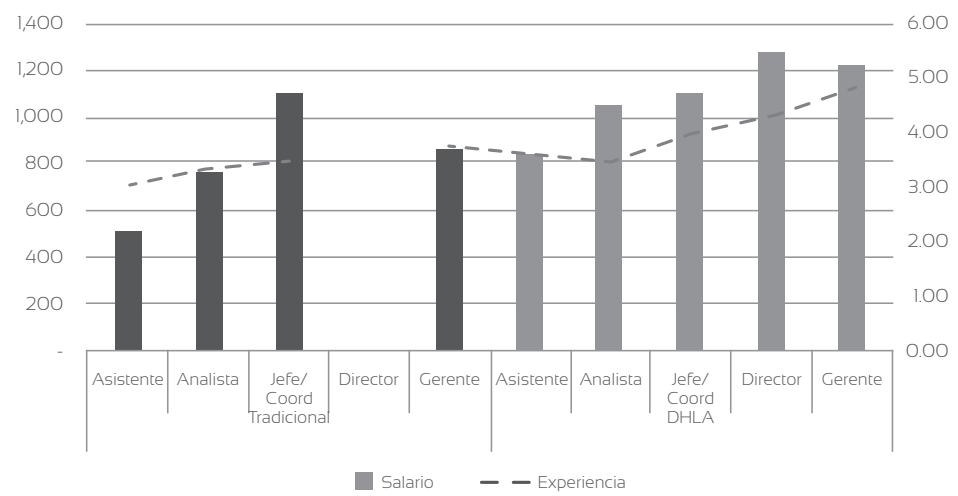

Figura 2. Salario promedio de los egresados del sistema dual y tradicional de la Universidad de Cuenca, por cargo y años de experiencia en el periodo 2010-2016 Fuente: elaboración propia. 
En la figura 3 se observa el género y el tiempo en que los egresados consiguieron empleo luego de graduarse, es decir, la inserción laboral. Se nota que el $63,10 \%$ y el $61,80 \%$ de los egresados mujeres y hombres de la dual, respectivamente, consiguen empleo antes de un mes, frente a un $43,80 \%$ y un $38,30 \%$ de mujeres y hombres, respectivamente, de la tradicional. También, un $2 \%$ de egresadas mujeres y $3 \%$ de egresados hombres de la dual consiguen empleo en un tiempo entre 7 o más de 12 meses, mientras que en ese mismo tiempo en la tradicional lo consiguen un $7,20 \%$ de mujeres y un 10,64 \% de hombres. Esto quiere decir, naturalmente, que los egresados de la tradicional tardan un mayor tiempo en la inserción laboral.

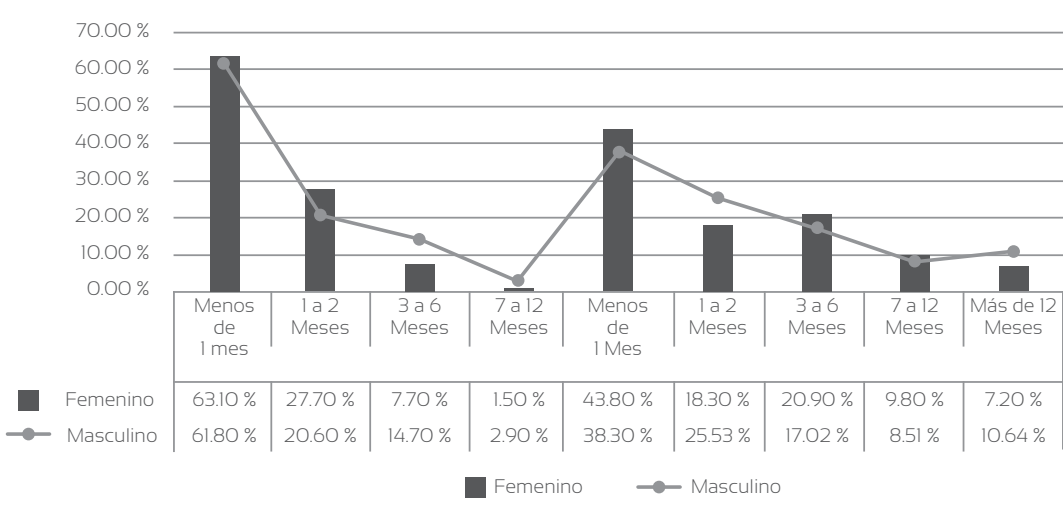

Figura 3. Datos comparativos de la inserción laboral promedio de los egresados de la modalidad dual y tradicional de la Universidad de Cuenca, por género en el periodo 2010-2016

Fuente: elaboración propia.

Con este primer análisis descriptivo y comparativo entre los grupos de tratamiento (dual) y de control (tradicional), el estudio se enfoca en evidenciar si dichas diferencias se mantienen. Para ello, la estimación de las puntuaciones de propensión para cada variable de tratamiento se realizó mediante un modelo probit, que aparece en el apéndice 1. Se efectuó un análisis previo de las correlaciones entre las variables explicativas y la variable dicotómica de impacto para garantizar que el programa dual no esté correlacionado con otras variables, controlando así otras características que pueden llevar a sesgos en su impacto. El resultado conforma el factor de inflación de la varianza (VIF, por su sigla en inglés) de 1,81.

Los resultados del cálculo del PSM se presentan en conjunto en la tabla 1. Se observa un pseudo $R^{2}$ igual a 0,0960, lo que indica que en este modelo la varianza total explicada de la probabilidad de participación es de un 9,60 \%. Además, se presenta la probabilidad o propensión de que un egresado 
participe en el programa dual mediante características observables (variables explicativas). En este caso, la probabilidad de que un egresado participe en el programa dual es menor si es mujer, casada con más experiencia, con un bachillerato en comercio/administración y ciencias exactas, si vive en la zona rural, proviene de la provincia del Azuay y trabaja en el sector de comercio. Por el contrario, ser bachiller en ciencias sociales, poseer una vivienda propia tipo casa, villa o media agua, trabajar en el sector de servicios y tener cargos de analista, gerente y jefe o coordinador de área, aumenta la probabilidad de que un egresado participe en el programa dual. Por consiguiente, se aprecia que los egresados con mejores condiciones económicas con especialización en ciencias sociales y con cargos ejecutivos tienen más probabilidades de participar en el programa dual, lo que se podría evidenciar que es un programa orientado a una población de estratos medios y medio altos.

Adicionalmente, se observa en los efectos marginales que las variables género, estado civil y sector donde laboran los egresados resultan ser estadísticamente significativos a un nivel del $5 \%, 1 \%$ y $5 \%$, respectivamente. Se encontró que una estudiante, al ser mujer y estar casada, reduce su probabilidad en 15 puntos porcentuales de pertenecer al programa dual; de igual forma, trabajar en el sector de servicios también disminuye su probabilidad en 14 puntos porcentuales de estar en el programa.

Según Hsieh y Hamilton (2016), y Tanaka, Tanaka y Kawakami (2015), en la estimación del PSM, se pueden incluir variables con bajo poder explicativo, para lograr la mayor calidad en el emparejamiento entre los grupos de tratamiento y control, en el que se busca que sean lo más semejantes posibles. Por otro lado, el bajo poder explicativo también podría ser atribuible a la presencia de otros factores no observables relevantes que afectan la decisión de participar en el programa, como pueden ser las habilidades, destrezas y competencias de los estudiantes.

\section{Tabla 1}

Resultado de la estimación PSM de pertenecer al programa dual, según características observables en el periodo 2010-2016

\begin{tabular}{ccc}
\hline \multicolumn{3}{c}{ Variable dependiente Programa Dual = 1} \\
\hline Variables explicativas / características & Coeficiente & Efecto marginal \\
\hline \multirow{2}{*}{ Género es femenino =1 } & $-0,289$ & $-0,149^{* *}$ \\
\hline \multirow{2}{*}{ Estado civil casado $=1$} & $(0,181)$ & $(0,067)$ \\
\hline
\end{tabular}


Variable dependiente Programa Dual = 1

\begin{tabular}{|c|c|c|}
\hline Variables explicativas / características & Coeficiente & Efecto marginal \\
\hline \multirow{2}{*}{ Experiencia (años) } & $-0,084 *$ & $-0,017$ \\
\hline & $(0,049)$ & $(0,017)$ \\
\hline \multirow{2}{*}{ Bachiller Comercio y Administración=l } & $-0,242$ & 0,075 \\
\hline & $(0,352)$ & $(0,124)$ \\
\hline \multirow{2}{*}{ Bachiller Ciencias Exactas = 1} & $-0,684^{*}$ & 0,145 \\
\hline & $(0,391)$ & $(0,156)$ \\
\hline \multirow{2}{*}{ Bachiller Ciencias Sociales = 1} & 0,156 & 0,081 \\
\hline & $(0,635)$ & $(0,252)$ \\
\hline \multirow{2}{*}{ Tipo vivienda casa o villa = 1} & 0,955 & 0,185 \\
\hline & $(0,619)$ & $(0,123)$ \\
\hline \multirow{2}{*}{ Tipo vivienda media agua = 1} & 0,985 & $-0,040$ \\
\hline & $(0,682)$ & $(0,210)$ \\
\hline \multirow{2}{*}{ Vive en la zona rural = 1} & $-0,411^{*}$ & $-0,011$ \\
\hline & $(0,231)$ & $(0,074)$ \\
\hline \multirow{2}{*}{ Proviene de la provincia del Azuay = 1} & $-0,451$ & 0,017 \\
\hline & $(0,326)$ & $(0,112)$ \\
\hline \multirow{2}{*}{ Trabaja sector comercial = 1} & $-0,355$ & $-0,012$ \\
\hline & $(0,235)$ & $(0,076)$ \\
\hline \multirow{2}{*}{ Trabaja sector de servicios $=1$} & 0,018 & $-0,138^{* *}$ \\
\hline & $(0,195)$ & $(0,068)$ \\
\hline \multirow{2}{*}{ Cargo analista =l } & $0,499 * * *$ & 0,043 \\
\hline & $(0,193)$ & $(0,068)$ \\
\hline \multirow{2}{*}{ Cargo gerente =1 } & $0,544 *$ & $-0,036$ \\
\hline & $(0,308)$ & $(0,105)$ \\
\hline \multirow{2}{*}{ Cargo jefe/coordinador =1 } & $0,807^{* * *}$ & 0,031 \\
\hline & $(0,216)$ & $(0,078)$ \\
\hline \multirow{2}{*}{ Constante } & $-0,323$ & \\
\hline & $(0,837)$ & \\
\hline LR chi2(15) & 37,81 & \\
\hline Prob > chi2 & 0,001 & \\
\hline Pseudo R2 & 0,0960 & \\
\hline Número de observaciones & 315 & \\
\hline
\end{tabular}

Nivel de significancia del ${ }^{* * *} 1 \%, * * 5 \%$ y $10 \%$

Nota: Regresión probit. La variable dependiente equivale a 1 si el egresado está en la dual y 0 en el caso contrario. Los coeficientes representan la contribución de cada variable explicativa/ característica a la probabilidad de que un egresado participe en el Programa dual.

Fuente: elaboración propia. 
Como ya se mencionó, los resultados del PSM buscan obtener un grupo de comparación (tradicional) que permita evaluar los efectos de la participación, y pretende que cada participante o varios no participantes tengan los mismos valores del PSM. Por tanto, dependen de que se disponga de una región considerable de soporte común en la distribución de las puntuaciones de propensión de las observaciones efectuadas en los dos grupos que se comparan. En este sentido, en la figura 4 se aprecia una región común de las distribuciones de las puntuaciones de propensión de los egresados a participar en los programas dual y tradicional; también, se ilustra que estas distribuciones se superponen perfectamente. Además, a lo largo de la zona de distribución, es relativamente fácil encontrar parejas porque egresados de la dual y tradicional comparten características similares. Sin embargo, los egresados de la dual con propensiones cercanas a 1 no pueden corresponderse con ningún egresado de la tradicional, ya que no existen con este nivel de puntuación (zona verde); se observa un mínimo rango no común en el extremo derecho de la distribución de las puntuaciones. La región común se localiza desde 0,058 hasta 0,723 ; el número de bloques de balanceo es 4, lo que representa un emparejamiento aceptable y apropiado, según lo determinado por Heinrich, Maffioli y Vázquez (2010); Dehejia y Wahba (2016), y Wagner, Newman y Javitz (2017).

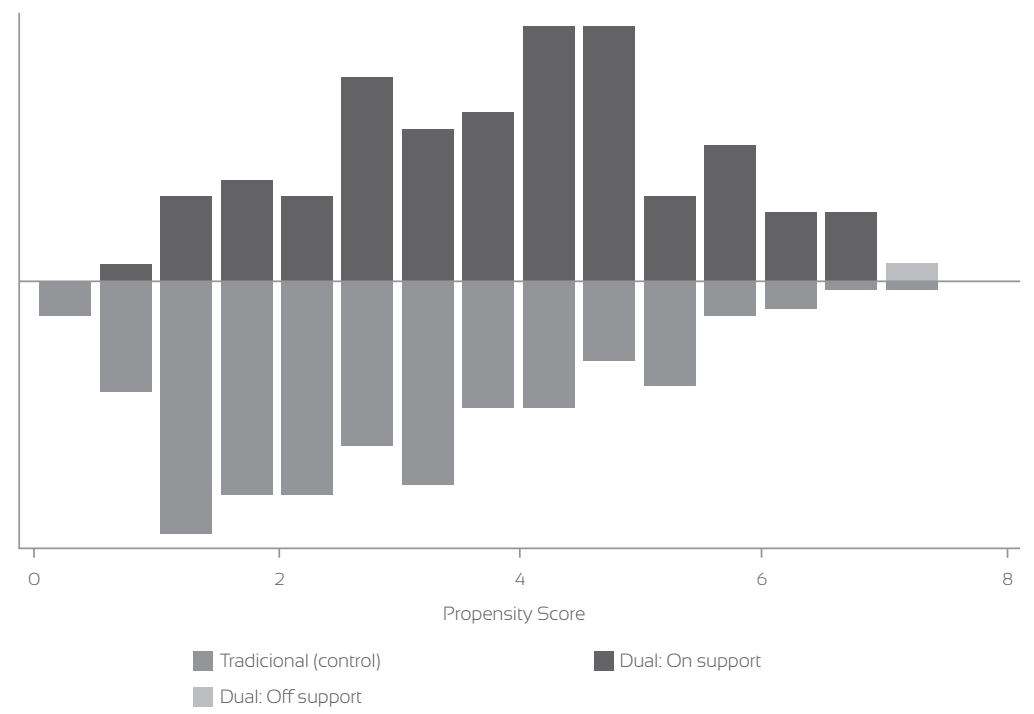

Figura 4. Región común estimación PSM a participar en el programa periodo 2010-2016 Fuente: elaboración propia. 
Tabla 2

Resultados ATET programa dual: salario y tiempo de inserción laboral (comparación de medias) en el periodo 2010-2016

\begin{tabular}{|c|c|c|c|c|c|c|}
\hline \multirow[b]{2}{*}{ Técnica } & \multirow[b]{2}{*}{$\begin{array}{c}\mathrm{N} . \\
\text { Tratamiento }\end{array}$} & \multirow[b]{2}{*}{$\begin{array}{c}\mathrm{N} . \\
\text { Control }\end{array}$} & \multicolumn{2}{|c|}{ Salario } & \multicolumn{2}{|c|}{ Inserción laboral } \\
\hline & & & atet & $\mathbf{t}$ & atet & $\mathbf{t}$ \\
\hline $\begin{array}{l}\text { Vecino más } \\
\text { cercano }\end{array}$ & 100 & 86 & $\begin{array}{c}226,11 \\
(53,024)\end{array}$ & $4,264^{* * *}$ & $\begin{array}{l}-2,917 \\
(0,747)\end{array}$ & $-3,905 * * *$ \\
\hline Radio matching & 100 & 211 & $\begin{array}{c}305,15 \\
(42,710)\end{array}$ & $7,145 * * *$ & $\begin{array}{c}-2,621 \\
(0,428)\end{array}$ & $-6,130 * * *$ \\
\hline Kernel matching & 100 & 211 & $\begin{array}{c}265,52 \\
(44,461)\end{array}$ & $5,972^{* * *}$ & $\begin{array}{l}-2,686 \\
(0,451)\end{array}$ & $-5,955 * * *$ \\
\hline
\end{tabular}

Significancia del *** $1 \%, * * 5 \%$ y $10 \%$.

Fuente: elaboración propia.

En el apéndice 2 se aprecia el efecto promedio de recibir el programa dual sobre los salarios o el tiempo de inserción en el mercado laboral de los egresados. De esta manera, en la tabla 2 se considera un ATET positivo y significativo al $1 \%$ en los tres métodos, considerando un efecto promedio significativo sobre los salarios de los egresados que recibieron el programa dual. Además, se observa una mayor robustez en la técnica radio matching, que muestra que los egresados que recibieron el programa dual mejoran sus salarios en promedio USD 305,15 más con respecto a los que no recibieron el programa, esto representa un $44 \%$ más con respecto al salario promedio de los egresados de la tradicional. De la misma manera, con la técnica de vecino más cercano y kernel matching se evidencia que constituye un $32 \%$ y un $38 \%$ más con respecto al salario promedio de un egresado de la tradicional, estamos ante una variación pequeña en relación con la primera técnica.

En lo referente al tiempo que toma un egresado en insertarse en el mercado laboral, se aprecia también un ATET con las tres técnicas que son estadísticamente significativas al $1 \%$. Para este caso, se considera que radio matching presenta una mayor robustez, lo que expresa que aquellos egresados que recibieron el programa dual les toma en promedio 2 meses 18 días insertarse en el mercado laboral, luego de egresar de la universidad. Esto significa que a un egresado de la dual le tomará insertarse un $65 \%$ del tiempo más rápido que a un egresado de la tradicional. La técnica del vecino más cercano y kernal matching muestran una significancia con una menor robustez. 
El apéndice 3 evalúa también el impacto estimado mediante el enfoque de puntuación de regresión, que computó primero individualmente el impacto para cada egresado inscrito en el programa dual y luego el promedio de esos impactos individuales, controlando de esta manera las diferencias en las características observables entre los dos grupos. Por tanto, en la tabla 3 se muestra el efecto medio significativo de tratamiento (programa dual) estimado sobre los salarios y el tiempo que tardan en insertarse al mercado laboral sus egresados, siendo USD 275,00 y en 2 meses 20 días, respectivamente.

De esta forma, el promedio de impacto de los salarios de los egresados de la dual aumentaría con los años de experiencia y los cargos jerárquico de gerente, director y coordinador desempeñados en las empresas, es decir, a medida de que una persona tenga un mayor número de años de escolaridad, su productividad también será mayor, situación que se verá reflejada con mejores puestos y mayores remuneraciones, pues los ingresos son iguales a su productividad marginal. Asimismo, la disminución del tiempo de inserción laboral estaría determinado por el sector de servicios y los cargos jerárquicos como analista y gerente, lo que confirma las observaciones realizadas sobre la base de las estadísticas descriptivas en las figuras 2 y 3 . Esto denota que los egresados duales tendrían una vinculación adecuada al mercado de trabajo determinado por el sector de servicios, que les permite ocupar cargos de mayor responsabilidad en la medida en que adquieren más experiencia.

Por último, se evidencia que las estimaciones de impacto del programa dual son sólidas para los diferentes enfoques de estimación expuestos en la tabla 2 y 3, proporcionando un ATET sobre los salarios y tiempo de inserción de sus egresados similares y notables.

\section{Tabla 3}

Resultados de impacto del programa dual: salario y tiempo inserción laboral (análisis puntuaciones de regresión) periodo 2010-2016

\begin{tabular}{lcccc}
\hline \multicolumn{1}{c}{ Variables dependientes: } & \multicolumn{2}{c}{ Salario } & $\begin{array}{c}\text { Tiempo de } \\
\text { inserción }\end{array}$ \\
\cline { 2 - 5 } & Coeficiente & P valor & Coeficiente & P valor \\
\hline programa $=1$ & $275.002^{* * *}$ & 0.000 & $-2.668^{* * *}$ & 0.000 \\
& $(34.672)$ & & $(0.476)$ & \\
\hline Género es femenino =1 & -28.76385 & 0.432 & -0.318 & 0.526 \\
& $(36.527)$ & & $(0.502)$ & \\
\hline Estado civil casado $=1$ & -0.474 & 0.989 & -0.302 & 0.506 \\
\hline Experiencia (años) & $(32.987)$ & & $(0.453)$ & \\
\hline
\end{tabular}




\begin{tabular}{|c|c|c|c|c|}
\hline \multirow[t]{2}{*}{ Variables dependientes: } & \multicolumn{3}{|c|}{ Salario } & \multirow{2}{*}{$\begin{array}{r}\begin{array}{c}\text { Tiempo de } \\
\text { inserción }\end{array} \\
\text { P valor }\end{array}$} \\
\hline & Coeficiente & Pvalor & Coeficiente & \\
\hline & (9.996) & & $(0.137)$ & \\
\hline \multirow{2}{*}{$\begin{array}{l}\text { Bachiller comercioComercio y } \\
\text { administraciónAdministración=1 }\end{array}$} & -108.992 & 0.126 & 0.310 & 0.751 \\
\hline & (71.036) & & (0.975) & \\
\hline \multirow[t]{2}{*}{$\begin{array}{l}\text { Bachiller ciencias } \\
\text { exactasCiencias Exactas }=1\end{array}$} & -92.043 & 0.234 & -0.215 & 0.839 \\
\hline & $(77.162)$ & & $(1.059)$ & \\
\hline \multirow{2}{*}{$\begin{array}{l}\text { Bachiller ciencias } \\
\text { socialesCiencias Sociales }=1\end{array}$} & -150.690 & 0.226 & -1.351 & 0.429 \\
\hline & $(124.226)$ & & $(1.706)$ & \\
\hline \multirow[t]{2}{*}{ Tipo vivienda casa o villa = 1} & 137.326 & 0.139 & -0.688 & 0.589 \\
\hline & (92.500) & & $(1.270)$ & \\
\hline \multirow[t]{2}{*}{ Tipo vivienda media agua $=1$} & 100.901 & 0.353 & -0.703 & 0.637 \\
\hline & $(108.424)$ & & $(1.489)$ & \\
\hline \multirow[t]{2}{*}{ Vive en la zona rural = 1} & $-74.507^{*}$ & 0.085 & -0.749 & 0.208 \\
\hline & $(43.180)$ & & (0.593) & \\
\hline \multirow[t]{2}{*}{$\begin{array}{l}\text { Proviene de la provincia del } \\
\text { Azuay }=1\end{array}$} & 80.423 & 0.217 & -1.151 & 0.198 \\
\hline & (64.944) & & $(0.892)$ & \\
\hline \multirow[t]{2}{*}{ Trabaja sector comercial = 1} & -48.858 & 0.285 & -0.050 & 0.937 \\
\hline & (45.635) & & $(0.627)$ & \\
\hline \multirow[t]{2}{*}{ Trabaja sector de servicios $=1$} & 18.504 & 0.639 & $0.993^{*}$ & 0.068 \\
\hline & (39.443) & & $(0.542)$ & \\
\hline \multirow[t]{2}{*}{ Cargo analistaAnalista =1 } & $171.089^{* * *}$ & 0.000 & $-1.536 * * *$ & 0.004 \\
\hline & (38.175) & & $(0.524)$ & \\
\hline \multirow[t]{2}{*}{ Cargo directorDirector =1 } & $332.413^{* *}$ & 0.046 & 2.375 & 0.297 \\
\hline & $(165.657)$ & & $(2.274)$ & \\
\hline \multirow[t]{2}{*}{ Cargo gerenteGerente $=1$} & $319.218^{* * *}$ & 0.000 & $7.144^{* * *}$ & 0.000 \\
\hline & (62.330) & & (0.856) & \\
\hline \multirow[t]{2}{*}{$\begin{array}{l}\text { Cargo jefe/coordinadorjefe / } \\
\text { Coordinador =l }\end{array}$} & $391.631^{* * *}$ & 0.000 & -0.243 & 0.688 \\
\hline & $(44.006)$ & & $(0.604)$ & \\
\hline \multirow[t]{2}{*}{ Constante } & 345.618 & 0.018 & 6.194 & 0.002 \\
\hline & (145.792) & & $(2.002)$ & \\
\hline Prob $>$ F & 0.0000 & & 0.0000 & \\
\hline R-squared & 0.4641 & & 0.3124 & \\
\hline $\begin{array}{l}\text { Numero de } \\
\text { observacionesObservaciones }\end{array}$ & 318 & & 318 & \\
\hline
\end{tabular}

Fuente: elaboración propia. 
Finalmente, estos resultados concuerdan con las investigaciones planteadas por Rojas (2015) y Segura (2016), que concluyeron que el salario y la inserción son factores relevantes y significativos en la formación dual en la Fundación Empresarial de la Cámara de Comercio de Bogotá y Colombia.

\section{Discusión}

Esta investigación, al cumplirse ya una década de inicio del programa educativo en la ciudad de Cuenca y en el país, contribuye a la generación de evidencia empírica para resaltar el impacto del programa sobre las variables salario y el tiempo que le toma al egresado insertarse en el mercado laboral. Los resultados en los dos enfoques muestran que existen diferencias significativas entre el grupo formado con la modalidad dual y el grupo formado con la modalidad tradicional.

La afirmación precedente se basa corrobora lo planteado por Zhibin y Weiping (2017), que la formación dual es un baluarte que mejora las condiciones de vida de sus profesionales, les permite una rápida incorporación al mercado laboral con una atractiva retribución económica. En consecuencia, la inserción laboral de los egresados duales estaría muy ligada al ajuste entre la formación recibida, la experiencia desarrollada en todo el proceso formativo y las exigencias del mercado de trabajo; en ella se despliega un puente entre la formación y el trabajo como un proceso continuo íntimamente ligado.

La investigación también demuestra que el modelo dual, como una modalidad de formación profesional y educativa, está creado para que los estudiantes adquieran un adiestramiento laboral mejorando sus ingresos económicos como se evidencia en la tabla 3. Esto concuerda con lo mencionado por Dang (2016), quien analizó los efectos de la formación en educación vocacional (VET) en el salario de los empleados en el contexto vietnamita y comprobó que el adiestramiento en el trabajo se asoció positivamente con los aumentos salariales.

También se aprecia que la formación dual ofrece una experiencia laboral temprana al estudiante, que se traduce en mayor salario y experiencia profesional, una ventaja competitiva frente a la formación tradicional. Coincide con lo citado por Becker (1975), al exponer que en el modelo de capital humano existe una relación directa entre los sueldos, la escolaridad y la experiencia en el trabajo (como se observa en la tabla 3). El tipo de formación que se brinda de este modo ayudaría a incluir esos elementos fundamentales de la educación que se le reclaman, como vimos, incorporar a la universidad: el aprendizaje significativo (al aprendizaje se vitaliza, tareas cuando encuentra asidero en las práctica de la 
empresa), tareas autónomas de enseñanza dirigidas a los estudiantes (las que gana en su labor empresarial) y recuperar las sinergias que ocurren en el mundo empresarial.

La investigación, igualmente, nos permite corroborar que las empresas son actores relevantes de la sociedad, y constituyen un viaducto entre el sistema educativo y la realidad productiva. De esta manera, coincidiendo con lo propuesto por Villarroel et al. (2017); Brunet y Rodríguez-Soler (2017), y Carvajal, Romero y Álvarez (2017), suponemos que la generación de conocimientos tecnológicos e innovadores que desarrollan y aplican las empresas son factores que los centros educativos superiores precisan adoptar y transferir a los estudiantes para una correcta inserción al mundo laboral.

La investigación muestra una alta participación de la mujer en los dos programas educativos; ellas laboran esencialmente en el sector de servicios y comercio, y sus salarios son un $10 \%$ y $18 \%$ menores con respecto al de los hombres. Los datos confirman lo mencionado por Chávez et al. (2017) sobre la falta de igualdad salarial entre ambos géneros y la discriminación que la mujer sufre en el mercado laboral. Es decir, ni siquiera el programa dual, que da cobijo a gente de clase media y alta, ha podido evitar este desequilibrio. Estamos entonces ante aspectos que aún deben trabajarse en el país.

Los buenos resultados obtenidos para el programa dual representan un mayor compromiso para la Universidad de Cuenca y el programa, pues la calidad de la educación debe ser una constante que caracterice el proceso educativo de la institución y que permita una buena proyección de sus egresados. En este sentido, los instrumentos de evaluación, la actualización de los planes, mallas curriculares deben ser objetivos para mejorar la calidad de los procesos educativos (Alemán, 2015 y Velázquez Albo, 2017). Las instituciones de educación superior deben, pues, mantenerse en constante innovación en sus procesos para crear y transferir capital humano en favor de una sociedad proyectista, para anticiparse a los cambios de la sociedad, enfrentarlos y estar así a la vanguardia de los cambios gubernamentales, tecnológicos, sociales, culturales, económicos. Para ello, haría bien si trabajasen de forma conjunta con las empresas o instituciones en las que laborarían los estudiantes para con esos datos, planes y expectativas contribuir al establecimiento de mallas y otros aspectos (Pilz, 2016; Pineda-Herrero, Argud-Morell y Ciraso-Calí, 2016).

En definitiva, Ecuador debería enfocarse a generar políticas públicas con una educación más profesionista como señala Bentolila, Cabrales y Jansen (2018), lo que generará en los egresados universitarios mayores competencias, habilidades y destrezas específicas que contribuirán a reducir los tiempos de inserción y adaptación en el mercado laboral. De esta forma, el modelo dual, como bien indicó Gómez (2017), constituye 
una innovadora alternativa de aprendizaje que, sin pretender sustituir al modelo de formación tradicional, supone una respuesta eficiente a los cambios globales.

Como limitaciones encontradas en este estudio, en primer lugar se destacan las pocas investigaciones llevadas a cabo en torno a la formación dual universitaria tanto en Ecuador como en Latinoamérica. De acuerdo con Kupffer (2015), esto obedece a que el modelo alemán no está contextualizado en su conjunto entre las instituciones educativas, sector productivo y los entes del Gobierno nacional y local. Es decir, se ha restado importancia a la inserción del modelo, lo que evita que se destaquen sus ventajas, que se repliquen modelos parecidos e incluso que los bachilleres conozcan esta oferta y la consideren seriamente para su formación. Dadas las ventajas del modelo, esta realidad termina por cuasi invisibilizar la alternativa, lo que terminar por afectar a la sociedad en general.

En segundo lugar, como sostuvieron Caliendo y Kopeinig (2008); Heinrich, Maffioli y Vázquez (2010); Imbens (2004); Hsieh y Hamilton (2016), y Tanaka, Tanaka y Kawakami (2015), el uso de las técnicas cuasiexperimentales Ileva implícitas ciertas limitaciones, ya que no consideran variables no observables o todos los factores que determinan que los egresados participen en el programa dual; existe siempre un sesgo de selección. Para superarlo, sugerimos otras investigaciones con métodos como diferencias en diferencias en años posteriores, asumiendo estos factores no observables que han recibido los egresados del modelo dual.

Finalmente, la investigación aporta evidencia del impacto del programa dual al asumir el salario y el tiempo que le toma al egresado insertarse en el mercado laboral, no podemos obviar lo indicado por Vera (2015) y Román, Franco y Camacho (2018): el proceso de inserción laboral de los egresados universitarios es multidimensional. Haría falta, entonces, incorporar otras competencias, habilidades y destrezas que el modelo propone y la empresa requiere para distinguir el efecto productividad que realmente posee en la educación y su efecto en el mercado laboral. Por lo pronto, los resultados preliminares que ofrecemos aquí parecen decirnos que el programa dual sí fortalece una serie de competencias necesarias en el mundo laboral, por eso la rápida empleabilidad de sus egresados. 


\section{Referencias}

Alemán, J. (2015). El sistema dual de formación profesional alemán: escuela y empresa. Educação e Pesquisa, 41(2), 495-511. Dol: http://doi. org/10.1590/s1517-97022015021532

Becker, G. (1975). Front matter, Human Capital: a theoretical and empirical analysis, with special reference to education. En Human Capital: A Theoretical and Empirical Analysis, with Special Reference to Education, Second Edition (pp. 22-0). Nueva York: Columbia University

Press. Recuperado de http://www.nber.org/chapters/c3730.pdf

Bentolila, S., Cabrales, A. y Jansen, M. (2018). El impacto de la FP dual sobre la inserción laboral de los jóvenes: Evidencia para la Comunidad de Madrid. Studies on the Spanish Economy, eee2018-20.

Berniell, L. y De la Mata, D. (2017). Prácticas laborales como mecanismo para mejorar la empleabilidad de los jóvenes: Lecciones para el caso argentino (No. 2017/28). Recuperado de http://scioteca.caf. com/bitstream/handle/123456789/1137/practicas-laborales-como-mecanismo-para-mejorar-la-empleabilidad-de-los-jovenes-lecciones-para-el-caso-argentino.pdf?sequence=1 \&isAllowed=y

Bodory, H., Camponovo, L., Huber, M. y Lechner, M. (2018). The finite sample performance of inference methods for propensity score matching and weighting estimators. Journal of Business \& Economic Statistics, 0015(May), 1-43. DOI: http://doi.org/10.1080/07350015.2018.1476247

Brunet Icart, I. y Rodríguez-Soler, J. (2017). The VET system and industrial SMEs: the role of employees with VET qualifications in innovation processes. Journal of Vocational Education and Training, 69(4), 596616. DOI: http://doi.org/10.1080/13636820.2017.1322130

Caliendo, M. y Kopeinig, S. (2008). Some Practical Guidance for the Implementation of Propensity Score Matching. Journal of Economic Surveys, 22(1), 31-72.

Carranza, A., Acosta, M. y Pulido, R. (2016). Modelo de formación dual del Tecnológico Nacional de México. Revista de Investigación En Educación, 14(2), 170-183.

Carvajal, R., Romero, A. y Álvarez, G. (2017). Estrategia para contribuir a la implementación de la formación dual de los profesionales de ciencias empresariales en las pequeñas y medianas empresas de la Provincia Tungurahua, Ecuador. Formación Universitaria, 10(5), 29-41. DOI: http://doi.org/10.4067/S0718-50062017000500004

Chavez Calle, L., Guzmán Solano, C., Rodríguez Jaramillo, K. y Toledo González, C. (2017). Autonomía financiera de la mujer frente al desarrollo sostenible: un desafío del siglo xxı. Gestión, Ingenio y Sociedad, 2(2), 104-109. Recuperado de http://gis.unicafam.edu.co/index.php/ gis/article/view/52 
Cordero Ferrera, J.M., Muniz Pérez, M. y Simancas Rodríguez, R. (2017). The relationship between cognitive and non-cognitive skills. Revista de Educación, (375), 36-60. Dol: http://doi.org/10.4438/1988-592XRE-2016-375-334

Dang, V. H. (2016). The Relationships Between the Vocational Education Training Providers and Enterprises: Theory and Practice. International Journal of Education and Literacy Studies, 4(2), 47-53. Dol: http://doi. org/10.7575/aiac.ijels.v.4n.2p.47

Dehejia, R. y Wahba, S. (2016). Propensity Score-Matching Methods for Nonexperimental Causal Studies. The MIT Press, 84(1), 15-161. Recuperado de http://www.uh.edu/ adkugler/Dehejia\&Wahba.pdf

Failla, V., Melillo, F. y Reichstein, T. (2017). Entrepreneurship and employment stability - Job matching, labour market value, and personal commitment. Journal of Business Venturing, 32(2), 162-177. DOI: http:// doi.org/10.1016/j.jbusvent.2017.01.002

Gessler, M. (2017). The Lack of Collaboration between Companies and Schools in the German Dual Apprenticeship System: Historical Background and Recent Data. International Journal for Research in Vocational Education and Training, 4(2), 164-195. DoI: http://doi.org/10.13152/ IJRVET.4.2.4

Gómez Galán, J. (2017). Nuevos estilos de enseñanza en la era de la convergencia tecno-mediática: hacia una educación holística e integral. IGERI, 8, 60-78. Recuperado de https://rio.upo.es/xmlui/handle/10433/5314

Heinrich, C., Maffioli, A. y Vázquez, G. (2010). A Primer for Applying Propensity-Score Matching. Washington: Inter-American Development Bank.

Herrera, F., Tinoco, G. y Guzmán, J. (2015). Modalidad de estudio dual, un nuevo modelo educativo en la formación de ingenieros. Revista Electrónica ANFEI digital, 2, 1-9.

Hsieh, M.-L. y Hamilton, Z. (2016). Predicting Success in Residential Substance Abuse Interventions: New Jersey's Pre-Release Incarceration Alternatives. Criminal Justice Policy Review, 27(2), 182-202. DOI: http://doi.org/10.1177/0887403414556148

Imbens, G. (2004). Nonparametric estimation of average treatment effects under exogeneity: A review. Review of Economics and Statistics, 86(1), 4-29.

Imbens, G. y Wooldridge, J. (2009). Recent developments in the econometrics of program evaluation. Journal of Economic Literature, 47(1), 5-86.

James, S., Roby, J., Powell, L., Teuscher, B., Hamstead, K. y Shafer, K. (2017). Does family reunification from residential care facilities serve children's best interest? A propensity-score matching approach in Ghana. 
Children and Youth Services Review, 83(October), 232-241. Dol: http:// doi.org/10.1016/j.childyouth.2017.10.032

Kenzhegaliyeva, M. (2018). German Dual System: A Model for Kazakhstan? Education in Modern Society, 16, 80-84.

Kool, A., Mainhard, T., Jaarsma, D., Van Beukelen, P. y Brekelmans, M. (2017). Effects of honours programme participation in higher education: a propensity score matching approach. Higher Education Research and Development, 36(6), 1222-1236. DOI: http://doi.org/10.1080/07294360 .2017.1304362

Kupffer, J. (2015). Formación profesional dual en Latinoamérica El sistema dual y su transferencia. La Paz. Recuperado de http://bolivien.ahk. de/fileadmin/ahk_bolivien/Publikationen/Studien/Kupffer_-_Transferencia_del_sistema_dual.pdf

López, Á., Virgüez, A., Sarmiento, J. y Silva, A. (2015). The Effect of Private Management of Public Schools on Student Achievement in Bogotá, Colombia. Ecos de Economía, 19(41), 108-136. Dol: http://doi. org/10.17230/ecos.2015.41.5

Lu, B., Cai, D. y Tong, X. (2018). Testing causal effects in observational survival data using propensity score matching design. Statistics in Medicine, 37(11), 1846-1858. Dor: http://doi.org/10.1002/sim.7599

Marhuenda-Luixá, F., Chisvert-Tarazona, M. J., Palomares-Montero, D. y Vila, J. (2017). Con "d" de "dual": investigación sobre la implantación del sistema dual a la formación profesional a España. Educar, 53(2), 285-307. DOI: http://doi.org/10.5565/rev/educar.821

Martín, A., Lope Peña, A., Carrasquer, P., Molina Romo, O., Moles, B. y Barrientos, D. (2017). Impacto de la formación en la empresa sobre las trayectorias laborales de los jóvenes: Definición del marco conceptual. Reflexiones en torno al reto de la formación en la empresa. (No. 21). QUIT Working Paper Series. Barcelona.

Medina, M. y Paniagua, R. (2017). Valoración multicriterio de la vinculación universidad-empresa en la UMSNH. Ethos Educativo, 50, 105-126.

Nasif, D., Vianco, A., Gertel, H., Flórez, J., Aguilera-Prado, M., Ruiz, J., ..., Hernández, M. (2017). Evaluación de la educación superior: una mirada desde Latinoamérica. Colección Educación Libros de Investigación. Cali, Colombia: Editorial Uniagustiniana. DOI: http://doi. org/10.28970/9789585639539

Orellana, M., Răileanu, M. y Argudo, D. (2016). A multilevel analysis of the returns to education in Ecuador. The multifaceted impact of human capital. Scientific Annals of Economics and Business, 63, 1-19. DOI: http://doi.org/10.1515/saeb-2016-0133

Ovalle, C. (2015). Sobre la técnica de puntajes de propensión (propensity score matching) y sus usos en la investigación en Educación. Educación y Ciencia, 4(43), 81-89. 
Pilz, M. (2016). Training Patterns of German Companies in India, China, Japan and the USA: What Really Works? International Journal for Research in Vocational Education and Training (IJRVET), 3(2), 66-87. DOI: http://doi.org/10.13152/IJRVET.3.2.1

Pineda-Herrero, P., Argud-Morell, I. y Ciraso-Calí, A. (2016). Factores que intervienen en la inserción laboral de los titulados en Educación en tiempos de crisis: un estudio sobre Cataluña. Revista de Educación, 372. DOI: 10.4438/1988-592X-RE-2015-372-318

Rivera, J. (2017). Los retos de la formación profesional: la formación profesional dual y la economía del conocimiento. Revista Internacional de Organizaciones, 17, 141-168. Dol: http://doi.org/10.17345/ rio17.141-168

Rojas., L. (2015). La formación dual en Colombia. El caso de la Fundación Universitaria de la Cámara de Comercio de Bogotá Uniempresarial: los desafíos actuales en la percepción de egresados y empresarios. Revista Científica General José María Córdova, 13(15), 145-181. Recuperado de http://www.scielo.org.co/pdf/recig/v13n15/v13n15a06.pdf

Román, J., Franco, T. y Camacho Solís, J. (2018). Inserción laboral de estudiantes de Gestión Turística de la Universidad Autónoma de Chiapas. Revista Global de Negocios, 6, 45-56.

Segura, J.C. (2016). Aportes para la medición del impacto de la política de formación para el trabajo: propuesta para la evaluación del contrato de aprendizaje. Finanzas y Política Económica, 8(2), 349-378.

Tanaka, S., Tanaka, S. y Kawakami, K. (2015). Methodological issues in observational studies and non-randomized controlled trials in oncology in the era of big data. Japanese Journal of Clinical Oncology, 45(4), 323-327. Dol: http://doi.org/10.1093/jjco/hyu220

Velázquez Albo, M. (2017). Tuning history in Latin America. Arts and Humanities in Higher Education, 16(4), 358-370. Dol: http://doi. org/10.1177/1474022216686522

Vera, M.J. (2015). En busca de lo inobservable: incorporando habilidades en un modelo á la Mincer (No. 2015/07). Venezuela: Banco de Desarrollo de América Latina - CAF.

Villarroel, C., Cabrales, F., Fernández, A. y Godoy, I. (2017). Indicadores de innovación y emprendimiento aplicados a la triple hélice en la Región de Arica y Parinacota, Chile. Interciencia, 42(11), 719-726.

Wagner, M., Newman, L. y Javitz, H. (2017). Vocational Education Course Taking and Post-High School Employment of Youth with Emotional Disturbances. Career Development and Transition for Exceptional Individuals, 40(3), 132-143. Dol: http://doi.org/10.1177/2165143415626399 
Yahaya, N., Sattar, M. y Mohamad, R. (2017). Exploring the Authentic Teaching and Learning Experience of the Apprentices of the National Dual Training System. International Journal of Academic Research in Business and Social Sciences, 7(3), 690-703. Dol: http://doi.org/10.6007/ IJARBSS/v7-i3/2021

Zhibin, T. y Weiping, S. (2017). On the Logic and Process of Collaborative Innovation in Higher Vocational Education and Industrial Development. Chinese Education and Society, 50(5-6), 458-468. Dol: http:// doi.org/10.1080/10611932.2017.1408327 


\section{Apéndice 1}

\section{Estimation of the Propensity Score}

$\begin{array}{llr}\text { Probit regression } & \text { Number of obs } & =315 \\ & \text { LR chi2(15) } & =37.81 \\ \text { Log likelihood }=-177.95178 & \text { Prob }>\text { chi2 } & =0.0010 \\ & \text { Pseudo R2 } & =0.0960\end{array}$

\begin{tabular}{r|rrrrrr}
\hline programa & Coef. & Std. Err. & $\mathrm{z}$ & $\mathrm{P}\rangle|\mathrm{z}|$ & [95\% Conf. Interval] \\
\hline genero & -.2895735 & .1814776 & -1.60 & 0.111 & -.6452631 & .0661161 \\
estado & -.1978613 & .1689578 & -1.17 & 0.242 & -.5290125 & .1332899 \\
experiencia & -.0847711 & .0498128 & -1.70 & 0.089 & -.1824023 & .0128602 \\
bachiller1 & -.2422649 & .3528455 & -0.69 & 0.492 & -.9338293 & .4492996 \\
bachiller2 & -.6845591 & .3916062 & -1.75 & 0.080 & -1.452093 & .0829751 \\
bachiller3 & .1566778 & .6350562 & 0.25 & 0.805 & -1.08801 & 1.401365 \\
vivienda1 & .955503 & .6197494 & 1.54 & 0.123 & -.2591835 & 2.170189 \\
vivienda3 & .9858973 & .6827974 & 1.44 & 0.149 & -.352361 & 2.324156 \\
zona & -.4119857 & .2311459 & -1.78 & 0.075 & -.8650233 & .0410518 \\
provincia1 & -.451617 & .3265157 & -1.38 & 0.167 & -1.091576 & .1883419 \\
sector1 & -.3559422 & .2351231 & -1.51 & 0.130 & -.816775 & .1048906 \\
sector3 & .0188536 & .1950471 & 0.10 & 0.923 & -.3634316 & .4011389 \\
posicion1 & .4992617 & .1931411 & 2.58 & 0.010 & .1207122 & .8778113 \\
posicion4 & .5442137 & .3089574 & 1.76 & 0.078 & -.0613317 & 1.149759 \\
posicion5 & .8072183 & .2162063 & 3.73 & 0.000 & .3834618 & 1.230975 \\
cons & -.3235766 & .8374527 & -0.39 & 0.699 & -1.964954 & 1.3178 \\
\hline
\end{tabular}

Note: the common support option has been selected

The region of common support is [.05801802, .72393595] 


\section{Apéndice 2}

ATT Estimation with Nearest Neighbor Matching Method

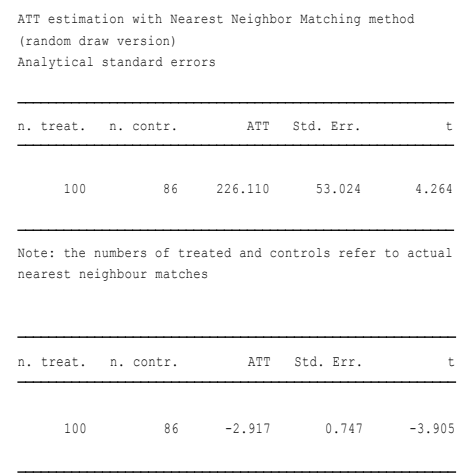

ATT Estimation with Radius Matching Method

\begin{tabular}{|c|c|c|c|c|}
\hline n. treat. & n. contr. & ATT & Std. Err. & \\
\hline 100 & 211 & 305.150 & 42.710 & 7.145 \\
\hline \multicolumn{5}{|c|}{$\begin{array}{l}\text { Note: the numbers of treated and controls refer to actual } \\
\text { matches within radius }\end{array}$} \\
\hline n. treat. & n. contr. & ATT & Std. Err. & $\mathrm{t}$ \\
\hline 100 & 211 & -2.621 & 0.428 & -6.130 \\
\hline
\end{tabular}

ATT Estimation with the Kernel Matching Method

ATT estimation with the Kernel Matching method

Bootstrapped standard errors

\begin{tabular}{lrrrr}
\hline n. treat. & n. contr. & ATT & Std. Err. & t \\
& & & & \\
100 & 211 & 265.521 & 47.112 & 5.636 \\
& & & & \\
\hline n. treat. & n. contr. & ATT & Std. Err. & \\
\hline & & & & \\
100 & 211 & -2.686 & 0.451 & -5.955 \\
& & & & \\
\hline
\end{tabular}




\section{Apéndice 3}

\section{Impacto sobre el salario medio del egresado}

\begin{tabular}{r|crc} 
Source & SS & df & MS \\
\hline Model & 18955010.9 & 17 & 1115000.64 \\
Residual & 21885298.2 & 300 & 72950.9941 \\
\hline Total & 40840309.1 & 317 & 128833.783
\end{tabular}

$$
\begin{array}{ll}
\text { Number of obs } & =318 \\
\mathrm{~F}(17, \quad 300) & =15.28 \\
\text { Prob }>\mathrm{F} & =0.0000 \\
\text { R-squared } & =0.4641 \\
\text { Adj R-squared } & =0.4338 \\
\text { Root MSE } & =270.09
\end{array}
$$

\begin{tabular}{r|rrrrrr}
\hline salario & Coef. & Std. Err. & $t$ & $\mathrm{P}\rangle|\mathrm{t}|$ & [95\% Conf. Interval] \\
\hline programa & 275.0023 & 34.67173 & 7.93 & 0.000 & 206.7717 & 343.2329 \\
genero & -28.76385 & 36.52719 & -0.79 & 0.432 & -100.6458 & 43.11811 \\
estado & -.4741556 & 32.98654 & -0.01 & 0.989 & -65.38846 & 64.44015 \\
experiencia & 40.16018 & 9.996381 & 4.02 & 0.000 & 20.48827 & 59.83209 \\
bachiller1 & -108.9924 & 71.03645 & -1.53 & 0.126 & -248.7852 & 30.80045 \\
bachiller2 & -92.04337 & 77.16229 & -1.19 & 0.234 & -243.8913 & 59.80453 \\
bachiller3 & -150.6899 & 124.2258 & -1.21 & 0.226 & -395.1542 & 93.77438 \\
vivienda1 & 137.3256 & 92.50038 & 1.48 & 0.139 & -44.70622 & 319.3573 \\
vivienda3 & 100.9009 & 108.4239 & 0.93 & 0.353 & -112.4668 & 314.2686 \\
zona & -74.50744 & 43.18014 & -1.73 & 0.085 & -159.4818 & 10.46689 \\
provincia1 & 80.4233 & 64.94394 & 1.24 & 0.217 & -47.38008 & 208.2267 \\
sector1 & -48.85826 & 45.63537 & -1.07 & 0.285 & -138.6642 & 40.94772 \\
sector3 & 18.50413 & 39.44258 & 0.47 & 0.639 & -59.11505 & 96.1233 \\
posicion1 & 171.0886 & 38.17532 & 4.48 & 0.000 & 95.96333 & 246.214 \\
posicion3 & 332.4127 & 165.6573 & 2.01 & 0.046 & 6.41522 & 658.4102 \\
posicion4 & 319.2178 & 62.33033 & 5.12 & 0.000 & 196.5577 & 441.8778 \\
posicion5 & 391.6308 & 44.00577 & 8.90 & 0.000 & 305.0317 & 478.2299 \\
\hline cons & 345.6179 & 145.7916 & 2.37 & 0.018 & 58.71417 & 632.5216 \\
\hline
\end{tabular}




\section{Impacto sobre el tiempo inserción medio del egresado}

\begin{tabular}{r|crr} 
Source & SS & df & MS \\
\hline Model & 1874.03818 & 17 & 110.23754 \\
Residual & 4125.44925 & 300 & 13.7514975 \\
\hline Total & 5999.48742 & 317 & 18.9258278
\end{tabular}

$\begin{array}{lr}\text { Number of obs } & =318 \\ \mathrm{~F}(17, \quad 300) & =8.02 \\ \text { Prob }>\mathrm{F} & =0.0000 \\ \text { R-squared } & =0.3124 \\ \text { Adj R-squared } & =0.2734 \\ \text { Root MSE } & =3.7083\end{array}$

\begin{tabular}{r|rrrrrr}
\hline mctiempo & Coef. & Std. Err. & $t$ & $\mathrm{P}>|\mathrm{t}|$ & [95\% Conf. Interval] \\
\hline programa & -2.66762 & .4760306 & -5.60 & 0.000 & -3.604402 & -1.730838 \\
genero & -.3180548 & .5015054 & -0.63 & 0.526 & -1.304969 & .6688592 \\
estado & -.3015218 & .4528935 & -0.67 & 0.506 & -1.192772 & .5897287 \\
experiencia & -.0045226 & .1372468 & -0.03 & 0.974 & -.2746109 & .2655658 \\
bachiller1 & .3097568 & .9753054 & 0.32 & 0.751 & -1.60955 & 2.229063 \\
bachiller2 & -.2150325 & 1.059411 & -0.20 & 0.839 & -2.299851 & 1.869786 \\
bachiller3 & -1.351343 & 1.705576 & -0.79 & 0.429 & -4.707751 & 2.005066 \\
vivienda1 & -.6875157 & 1.269998 & -0.54 & 0.589 & -3.186748 & 1.811716 \\
vivienda3 & -.7032079 & 1.488622 & -0.47 & 0.637 & -3.632672 & 2.226256 \\
zona & -.7488003 & .5928481 & -1.26 & 0.208 & -1.915468 & .4178673 \\
provincia1 & -1.151011 & .8916574 & -1.29 & 0.198 & -2.905706 & .6036845 \\
sector1 & -.0498775 & .6265575 & -0.08 & 0.937 & -1.282882 & 1.183127 \\
sector3 & .9928512 & .5415327 & 1.83 & 0.068 & -.0728327 & 2.058535 \\
posicion1 & -1.535837 & .5241336 & -2.93 & 0.004 & -2.567281 & -.5043927 \\
posicion3 & 2.374563 & 2.274416 & 1.04 & 0.297 & -2.101267 & 6.850394 \\
posicion4 & 7.144015 & .8557734 & 8.35 & 0.000 & 5.459936 & 8.828094 \\
posicion5 & -.2428161 & .6041838 & -0.40 & 0.688 & -1.431791 & .946159 \\
cons & 6.19362 & 2.001667 & 3.09 & 0.002 & 2.254533 & 10.13271 \\
\hline & & & & & & \\
\hline & & & & & & \\
\hline
\end{tabular}

